\title{
A MOLECULAR CONCEPT OF MANAGING DATA
}

\author{
Christoph Schommer \\ Department of Computer Science, University Luxembourg, 6 Coudenhove-Kalergi, 1359 Luxembourg, Luxembourg
}

Keywords: Artificial life, Relational data management, Pattern recognition, Bio-inspired computing.

\begin{abstract}
The following (position) paper follows the concept of the field of Artificial Life and argues that the (relational) management of data can be understood as a chemical model. Whereas each data itself is consistent with atomic entities, each combination of data corresponds to a (artificial) molecular structure. For example, an attribute $D$ inside a relational system can be represented by a nucleus $\alpha_{D}$ sharing a cloud of values, which consists of so-called valectrons (the values for the column $D$ ). By using reaction rules like the selection of tuples or projection of attributes, a retrieve of molecules can be achieved quite easily. Advantages of the chemical model are no data types, a fast data access, and the associative nature of the molecules: this automatically supports a direct identification of patterns in the sense of data mining. A disadvantage is the need for restructuring that must eventually be done, because the incoming data stream is allowed to influence the chemical model. With this position paper, we present our basic concept.
\end{abstract}

\section{INTRODUCTION}

Since more than 30 years, relational database systems are successful, being the most important data management system worldwide. Based on the theory on sets, a relational database system takes advantage from the concepts of the relational algebra, which has led - among other functionalities - to today's standard query language SQL. Although in recent past, some alternative database architectures have been developed (object-oriented, object-relational, and XML databases, etc.), they have never received the desired breakthrough. And although the relational systems suffer from an efficient management (often, the more complex the system is the more time and capacity is needed to guarantee the data consistency), the relational architecture still proves reliability, consistency, and precision.

With this position paper, we foster on a completely different approach of data management and try to figure out that data is unlike a data value inside a well-structured environment but even more a fluid (dynamic) and molecular concern. With respect to the natural example, we understand data as an atomic structure and combinations of data as a molecule invoked on the field of Artificial chemistry (Dittrich et al., 2001). Commonly, Artificial chemistry is un- derstood as a theoretical model following the natural example, which is used to simulate types of systems in the spirit of chemical reactions (Leach, 2001). It originates in the field of Artificial Life (Kelemen and Sosík, 2001) and has proven to be a manifold and powerful pathway of modeling (Skusa et al., 2000), (Ziegler and Banzhaf, 2001), (Schommer, 2009).

In general, an artificial chemistry is defined as a triple $(M, R, A)$, where $M$ refers to the set of molecules $\left\{\mathrm{m}_{1} \ldots, \mathrm{m}_{n}\right\}$, which is possibly of infinite size, $\mathrm{R}$ to the a set of n-ary operations/reaction rules $\left\{\mathrm{r}_{1} \ldots, \mathrm{r}_{n}\right\}$ on the molecules, and $\mathrm{A}$, which denotes an algorithm describing how to apply the rules $\mathrm{R}$ to a subset $\mathrm{P} \subset \mathrm{M}$. Each reaction rule $\mathrm{r}_{i} \in \mathrm{R}$ is written as a chemical reaction like

$$
\left(x_{1}, x_{2}\right) \rightarrow^{r_{i}} x_{1}^{*} x_{2}^{*}
$$

With that, we firstly introduce the molecular model, present several reaction rules to explain its depth, and demonstrate its strength on an example.

\section{A SET OF MOLECULES}

We understand each attribute $D_{i}$ inside a database table $D$ as a nucleus $\alpha_{i}$ that owns a cloud of values $e_{1}, \ldots e_{k}$ at distance $\varepsilon_{i}$. Each $e_{i}$ corresponds to a data 
value $d_{i} \in D_{i}$ that might be e.g. of type string or number (integer, real, dots), but not a list of values (first normal form is valid). A nucleus $\alpha_{i}$ owns a name (= the attribute name) and shares a higher valency $v_{D_{i}}$, the more dense the cloud of values is. The distance between the nucleus $\alpha_{i}$ and each valectron $e_{i}$ gives the strength of existence, meaning that if the occurrence of $e_{i}$ increases the occurrence of $e_{j}$, the distance to the nucleus is shorter. If the nucleus owns only one $e_{i}$, then $\alpha_{i}=e_{i}$.

a)

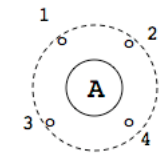

b)

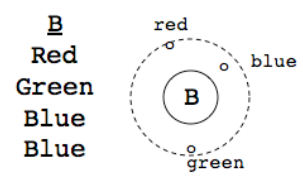

Figure 1: Database attributes $D_{A}$ and $D_{B}$ with the corresponding nuclei $\alpha_{A}$ and $\alpha_{B}$.

Figure 1 presents two database attributes $D_{A}$ and $D_{B}$ with the corresponding nuclei $\alpha_{A}$ and $\alpha_{B}$. Whereas all valectrons of nucleus $\alpha_{A}$ shares the same distance, the distance of $e_{b l u e}$ of nucleus $\alpha_{B}$ is shorter than for $e_{\text {green }}$ and $e_{\text {red }}$.

In opposite to its atomic basis, a database table of $\geq 2$ attributes $D_{1}, \ldots, D_{k}$ is consequently a set of nuclei $\alpha_{D_{1}}, \ldots, \alpha_{D_{k}}$. The nuclei, however, are not organised in an arbitrary way, but keep themselves ordered:

- The lower the valency $v_{D_{i}}$ is the more centric the nucleus $\alpha_{i}$ will be.

- In case that some nuclei share the same valency, we may randomly select one of them.

Figure 2: Simulation of two database attributes $D_{A}$ and $D_{B}$ of the database table $D$ with the corresponding threedimensional molecule $\mathrm{m}_{A, B}$ (ordered by their valency).

The principle of ordering is unlike the ordering of a set of numbers but more the arrangement of the nucleus including their cloud of values. With respect to this, a chemical structure of size $\geq 2$ which is said as to be a molecule $\mathrm{m} \in \mathrm{M}$ - therefore can not be a two-dimensional model anymore: the cloud of values embraces each previously selected nucleus and associates each valectron $e_{i}$ with its corresponding partner of the other nucleus. Figure 2 shows a simulation of two database attributes $D_{A}$ and $D_{B}$ of the database table $D$ with the corresponding three-dimensional molecule $\mathrm{m}_{A, B}$ (ordered by their valency). As presented in Figure 2, the merge of nuclei is as follows:

- Assume that $v_{D_{i}}<v_{D_{i+1}}<\ldots v_{D_{k}}$, then $v_{D_{i}}$ has the highest priority and therefore takes over the innermost position, followed by $v_{D_{i+1}}$, and so on.

- The nuclei $\alpha_{i}, \ldots \alpha_{k}$ are nested and represented by their cloud of values only.

- Originally associated tuples inside $\mathrm{D}=$ $\left\{D_{i}, \ldots D_{k}\right\}$ are connected by molecular bridges $\gamma_{i, k}$ of a certain strength, which may vary.

\section{ENZYMATIC REACTIONS}

An artificial enzyme is a protein that is able to execute reactions. Whereas in the natural example an enzyme takes over the responsibility of many functions that concern the metabolism of an individual, the simulation of enzyme in a database environment can be understood as the adequate to reaction rules. Enzymatic reactions work in one or two ways

- the targeted nuclei $\alpha_{i}$ can be copied.

- the molecular bridges $\gamma_{i, k}$ between the valectrons may be destroyed.

but the enzymatic instruction decides if both or only the latter action takes place. For example, an enzyme that simply has to read existing molecules surely copies the existent structure and then keeps only those connections that satisfy the enzymatic instruction. On the other side, a permanent delete of data in the original molecule does not afford a copy but only the delete of the molecular bridges.

With respect to a retrieval, fundamental enyzmes concern the selection and projection enzyme. Given a molecule as presented in Figure 2, then the reaction rule $\sigma_{A, B}$ characterizes a chemical reaction of the original molecule - which consists of the two cloud of values $\mathrm{A}$ and $\mathrm{B}$ - to another molecule $A^{*} B^{*}$. The density and the valency change, since for example $\mathrm{v}_{A, B}>\mathrm{v}_{A^{*} B^{*}}$ of the new molecule:

$$
A, B \rightarrow^{\sigma} A^{*} B^{*}
$$

Similarly, the reaction rule $\pi$ characterizes a chemical reaction as well, but in contrast to the reaction $\sigma$, the valency remains stable, whereas the number of resulting nuclei $\alpha_{i}$ changes:

$$
A, B \rightarrow{ }^{\pi} A^{*} B^{*}
$$


As an example, Figure 3 shows an enzymatic reaction $\pi_{B}\left(\sigma_{B=\text { 'blue }}(D)\right)$, where the original molecule is copied and all molecular bridges and valectrons (except 'blue') are removed. Please note that the distance of $\varepsilon_{\text {blue }}$ remains unchanged, i.e., the valectron remains at the same position.

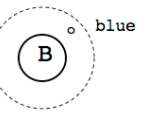

Figure 3: Examples of the Reactor Rule $\sigma$ : select B from D where $\mathrm{B}=$ 'Blue'

Similarly, Figure 4 shows an enzymatic reaction $\pi_{A}\left(\sigma_{A>2}(D)\right)$, where two valectrons occur.

$$
\text { (A) }
$$

Figure 4: Examples of the Reactor Rule $\sigma$ : select A fom D where $\mathrm{A}>2$

The enzymatic reaction $\pi_{A}\left(\sigma_{A>4}(D)\right)$ given in Figure 5, however, gives only the nucleus $\alpha_{A}$, but no valetrons.

\section{A}

Figure 5: Examples of the Reactor Rule $\sigma$ : select A from D where $\mathrm{A}>4$

Finally, Figure 6 shows an enzymatic reaction $\pi_{A, B}\left(\sigma_{A=2}(D)\right)$ where both nuclei $\alpha_{A}$ and $\alpha_{B}$ occur and the molecular bridge $\gamma_{\text {' } \text { green }^{\prime}, 2}$ between the corresponding valectrons still exist.

With an enzymatic reaction $\epsilon_{m, M}$, the insert of a new molecule $m$ into an existing molecular structure $\mathrm{M}$ takes place by a simple addition. In case that valectrons are already present, these become merged. As an example, Figure 7 shows the merge of two molecules where the valectron $e_{3}$ is common. However, such an insert may violate the correctness of the existing data landscape, because it allows the creation of molecules that do not exist. The insert of the molecule

$$
\epsilon_{\left(e_{5}, e_{6}, e_{3}, e_{4}, e_{9}\right), M}
$$

seems to be safe, but the existence of another molecule $\left(e_{1}, e_{2}, e_{3}, e_{7}, e_{8}\right)$ causes an error, since inherently the molecules $\left(e_{5}, e_{6}, e_{3}, e_{7}, e_{8}\right)$ and $\left(e_{1}, e_{2}\right.$, $\left.e_{3}, e_{4}, e_{9}\right)$ might untruly be present as well. By using just one molecular bridge $\gamma_{i, j}$, we therefore risk the inconsistency of the whole molecule.

An insert, and moreover the presence of collections of valectrons must not have a single molecular bridge but a double one. With this, the dashed bridge

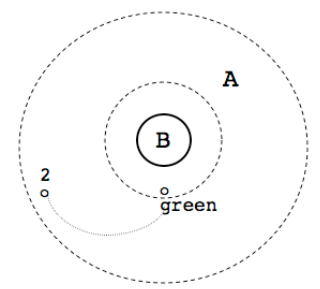

Figure 6: Examples of the Reactor Rule $\sigma$ : select B from D where $\mathrm{A}=2$

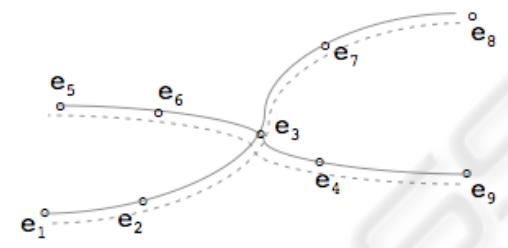

Figure 7: The reaction rule $\in(m, M)$ : a molecular bridge (dashed) characterizes the connections of the valectrons (= the $\beta$-helix $\beta_{e_{i} \ldots e_{e_{j}}}$ ) whereas the solid line the situation of the molecule after insertion $(\gamma$-helix).

characterizes the connections of the valectrons. This called the $\beta$-helix $\beta_{e_{i} \ldots e_{e_{j}}}$. The solid line the situation of the molecule while insertion, representing values between the associated nuclei $\alpha_{i}$. The molecular string is therefore called the $\alpha$-helix. And with that, a molecule $\left(e_{5}, e_{6}, e_{3}, e_{7}, e_{8}\right)$ does not exist since no $\alpha$-helix is from $e_{5}-e_{6}-e_{3}$ to $e_{7}$.

As a third operation, an (equi-)join operation of molecules may be represented by the reaction rule $\triangleleft_{=}\left(M_{i}, M_{j}\right)$. As for the insert reaction $\in$, those valectrons, which occur both in molecule $M_{i}$ and in $M_{j}$, are merged. All original valectrons keep their helix structure (see Figure 8).

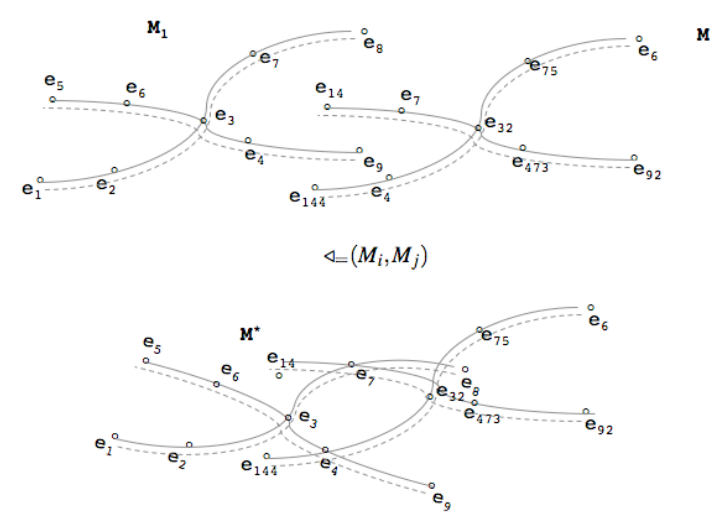

Figure 8: Reaction Rule $\triangleleft=\left(M_{i}, M_{j}\right)$

With an enzymatic reaction $\notin_{m, M}$, we denote the delete of a molecule $\mathrm{m}$ within M. The helix $\beta_{e_{i} \ldots e_{e_{j}}}$ guarantees that only those valectrons, which belong together, are deleted. 
In addition, the composition of several reaction rules like

$$
A, B \rightarrow^{\sigma, \pi} A^{*} B^{*}
$$

is possible and appears in that order the reaction rules are given. The composition is commutative.

Beside the given reaction rule, the authorization of valectrons might be interesting as well. With $a u$ thorization, we identify the an enzyme's right to access to a nuclei and it's cloud of values. This is not really a reaction rule as the enzymatic reaction does not results in a chemical reaction; it is more a feature of the nuclei itself that allows or disallows a permitted access. We therefore note a disallowed access by

$$
\neg \alpha_{A}
$$

meaning that the nucleus $\alpha_{A}$ rejects any kind of reaction. Instead of delivering a valectron, the result could be an empty element.

\section{DISCUSSION}

The idea of understanding data within an artificial chemical system is potentially unlike the relational system but offers a variety of characteristics. First, no data type specification is needed. The presence of a data item within the chemical database model is per se self-explaining and does not need any further specification concerning its type. The consequence then is that data (of different data type - from a relational point of view) is being identical. This is not of disadvantage because the expression of strength between valectrons through the molecular bridges $\gamma_{i, j}$ is very present. In fact, this is the second point as strong relationships among valectrons do inherently exist. If a combination of valectrons $e_{i}-e_{j}$ occurs often enough, then its molecular bridge $\gamma_{i, j}$ becomes stronger as if it occurs only "a few times". Third, the consideration of the molecular model towards a molecular-associative construct offers the identification of molecular clumps that are connected with each other and that represent a symbol, such that they may form a higher-related (cognitive) construct like a mental image or simply a thought. Assuming, that "tree" (for nucleus $\alpha_{1}$ ), "green" (for nucleus $\alpha_{2}$ ), and "rain" (for nucleus $\alpha_{3}$ ) exist, it would certainly be possible to think of a "staying in the forest on a cold and rainy day". As a last point, the molecular data management model as described above is open for the input of data streams. Whereas the relational model lacks from high administrative efforts, a stream of data may be handled more effective in the proposed model.

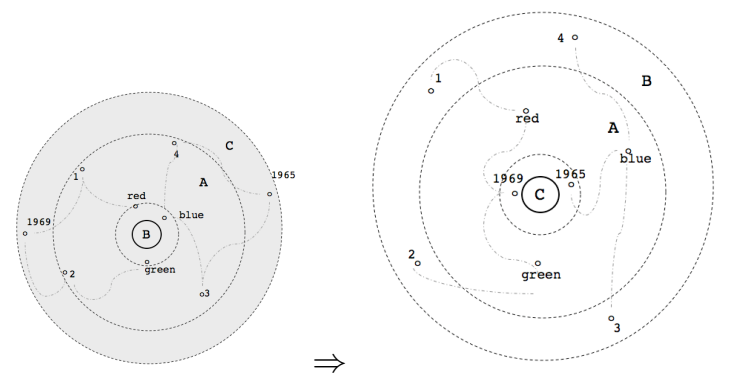

Figure 9: Restructuring the molecule: the left molecule refers to the situation where the number of years $\left(\alpha_{C}\right)$ is significantly less than the colour $\left(\alpha_{A}\right)$ and the amount of $\left(\alpha_{B}\right)$, whereas the right molecule refers to the more stable molecule.

On the other side, some kind of efforts is to be done in keeping the molecules in a stable and consistent form. Stability refers to a general claim that such nuclei $\alpha_{i}$ with a minor valency $v_{i}$ do more contribute to a general model consistency and therefore to the stability as well as those nuclei with a more densecloud of values. In consequence of a delete or an insert of molecules, a restructuring reaction must take place in order to guarantee stability and consistency. With respect to this, assume that an insert of a new data leads to a change of the valency with $v_{D_{i}}>v_{D_{i+1}}<\ldots v_{D_{k}}$. Then, the enzymatic restructuring $\psi$ is as follows:

- A copy $\alpha_{i}^{\prime}$ of the nuclei $\alpha_{i}$ is created; it is then set on its new place, depending on its valency $v_{D_{i}}$.

- All valectrons $e_{i}$ of $\alpha_{i}$ walk on the $\beta$-helix $\beta_{i}$ and finally reach their cloud of values.

- At each point, a connection of each valectron remains.

On the other side, a continuous change of the number of values may become counter-productive and finally refer to a continuous and repeating restructuring of the molecule, such that nuclei are more concerned with internal configurations than with the management of data. An alternative therefore is to prefer those nuclei whose cloud of values do not or even less changes in size. Once the molecule is created (first approach) and once a certain information about stable nuclei have been got, the second solution seems to be more appropriate.

\section{CONCLUSIONS}

With the presented proposition, we follow the concept of understanding data and information as an (artificial) chemical model. Each data is consistent with 
an atomic entity but each combination of data corresponds to a molecular structure. An attribute $D$ inside a relational system is represented as a nucleus $\alpha_{D}$ sharing a cloud of values, which consists of socalled valectrons. The nucleus satisfies the first normal form (atomic values). By using reaction rules like the selection of tuples $\sigma$ or projection of attributes $\pi$, a retrieve of molecules can be achieved quite easily. As mentioned in chapter 4, one of the major advantages is the associative nature of the molecule. The generation of mental images (or thoughts), beside the implementation of the given system, will then be next steps.

\section{ACKNOWLEDGEMENTS}

This work is currently been done within the International Laboratory for Intelligent and Adaptive Systems of the University of Luxembourg. We thank the members of the MINE research group for their support.

\section{REFERENCES}

Dittrich, P., Ziegler, J., and Banzhaf, W. (2001). Artificial chemistries-a review. Artificial Life, 7(3):225-275.

Fernández-Baizán, M. C., García, A., González, M. M., Pérez-Llera, C., Portaencasa, R., and Santos, E. (1996). Analysis and design of a relational database management system and implementation of its nucleus. Computers and Artificial Intelligence, 15(4).

Gerrilsan, R. (1975). The application of artificial intelligence of data base management. In IJCAI, pages 521527.

Hutton, T. J. (2002). Evolvable self-replicating molecules in an artificial chemistry. Artificial Life, 8(4):341-356.

Kelemen, J. and Sosík, P., editors (2001). Advances in Artificial Life, 6th European Conference, ECAL 2001, Prague, Czech Republic, September 10-14, 2001, Proceedings, volume 2159 of Lecture Notes in Computer Science. Springer.

Leach, A. (2001). Molecular Modelling - Principles and Applications. Prentice Hall, 2nd edition.

Schommer, C. (2009). An artificial molecular model to foster communities. In Knowledge Discovery and Information Retrieval (KDIR). IEEE Computer Society.

Skusa, A., Banzhaf, W., Busch, J., Dittrich, P., and Ziegler, J. (2000). Künstliche chemie. KI, 14(1):12-19.

Tominaga, K., Watanabe, T., Kobayashi, K., Nakamura, M., Kishi, K., and Kazuno, M. (2007). Modeling molecular computing systems by an artificial chemistry its expressive power and application. Artificial Life, 13(3):223-247. von Luck, K. and Marburger, H., editors (1994). Management and Processing of Complex Data Structures, Third Workshop on Information Systems and Artificial Intelligence, Hamburg, Germany, February 28 March 2, 1994, Proceedings, volume 777 of Lecture Notes in Computer Science. Springer.

Ziegler, J. and Banzhaf, W. (2001). Evolving control metabolisms for a robot. Artificial Life, 7(2):171-190. 